\title{
An overview of erosion-deposition studies for the JET Mk II high delta divertor*
}

\author{
A Widdowson ${ }^{1}$, S Brezinsek ${ }^{2}$, J P Coad ${ }^{1}$, D E Hole ${ }^{3}$, J Likonen ${ }^{4}$, \\ V Philipps ${ }^{2}$, M Rubel ${ }^{5}$, M F Stamp $^{1}$ and JET-EFDA contributors ${ }^{6}$ \\ ${ }^{1}$ EURATOM/UKAEA Fusion Association, Culham Science Centre, Abingdon OX14 3DB, UK \\ ${ }^{2}$ Institute of Energy Research, IEF-4: Plasma Physics, Forschungszentrum Jülich GmbH, \\ EURATOM Association, Trilateral Euregio Cluster, D-52425 Jülich, Germany \\ ${ }^{3}$ Department of Engineering and Design, University of Sussex, Brighton BN1 9QH, East Sussex, UK \\ ${ }^{4}$ Association EURATOM-Tekes, VTT, PO Box 1000, FI-02044 VTT, Finland \\ ${ }^{5}$ Royal Institute of Technology, Assoc. EURATOM-VR, 10044 Stockholm, Sweden \\ ${ }^{6}$ JET-EFDA, Culham Science Centre, Abingdon OX14 3DB, UK \\ E-mail: anna.widdowson@ccfe.ac.uk
}

Received 27 October 2009

Accepted for publication 5 November 2009

Published 30 December 2009

Online at stacks.iop.org/PhysScr/T138/014005

\begin{abstract}
Post-mortem analyses of tiles removed from the JET MkII HD divertor in 2007 are presented. The results indicate an increase in deposition at the outer plasma-shadowed region of the divertor, not seen prior to 2004 and indicate a shift away from the asymmetric picture of net deposition at the inner divertor compared with no overall deposition or erosion at the outer divertor. Surface analysis of the inner and outer vertical divertor tiles is largely the same as observed previously; however, a notable increase in Be composition on the inner and outer floor tiles is observed. An attempt has been made to correlate these data with campaignaveraged plasma configurations and spectroscopy results. While some changes in deposition/erosion characteristics can be explained, further detailed analysis of diagnostics and especially of time-resolved data, such as from rotating collector and quartz microbalance diagnostics, is required.
\end{abstract}

PACS numbers: 52.40.Hf, 52.55.Fa, 82.80.Yc

(Some figures in this article are in colour only in the electronic version.)

\section{Introduction}

Post-mortem analyses of tiles removed periodically from the JET vessel have established the current picture of asymmetric deposition/erosion characteristics between the inner and outer divertor legs. Such empirical data have provided information for the modelling of plasma surface interaction and material migration. This paper presents post-mortem analysis results for tiles removed from the JET vessel in 2007, thus providing the latest erosion and deposition data for further modelling studies.

Since 1995 sets of tiles forming a poloidal cross section have been taken from each of the divertor geometries; 1994-1995 MarkI (MkI), 1996-1998 MkIIA, 1999-2001

* See the appendix of Romanelli F et al 2008 Fusion Energy Conf. (Proc. 22nd FEC, Geneva, 2008) IAEA.
MkII Gas Box (MkII GB), 2002-2004 MkII Septum Replacement Plate (MkII SRP) and 2005-present MkII HD divertor, with the latest results reported here coming from tiles removed from the MkII HD divertor during the intervention of the JET vessel in 2007. Since the installation of the MkII GB divertor the vertical tiles (tiles 1, 3, 7 and 8) and floor tiles (tiles 4 and 6) in the divertor have remained the same, with the central section at the bottom of the divertor being the principal difference between geometries. Figure 1 shows these geometries along with the terminology used throughout this paper such as the plasma accessible and plasma-shadowed regions, which are indicated; tile numbers (1-8) are indicated on figure $1(\mathrm{c})$. Details of the earlier geometries (MkI and MkIIA) can be found elsewhere [1].

Early evidence for net deposition at the inner divertor compared with no overall deposition or erosion at the outer 


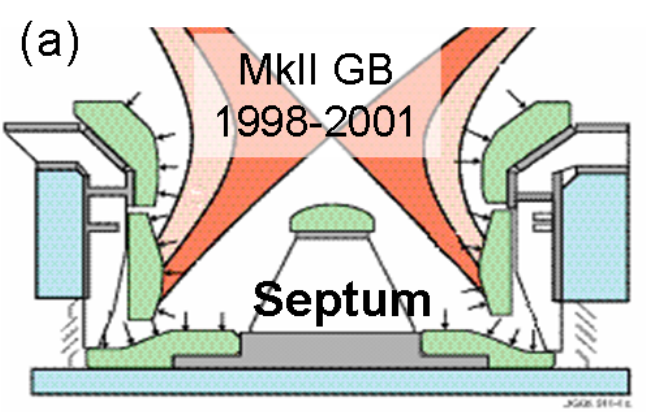

(b)
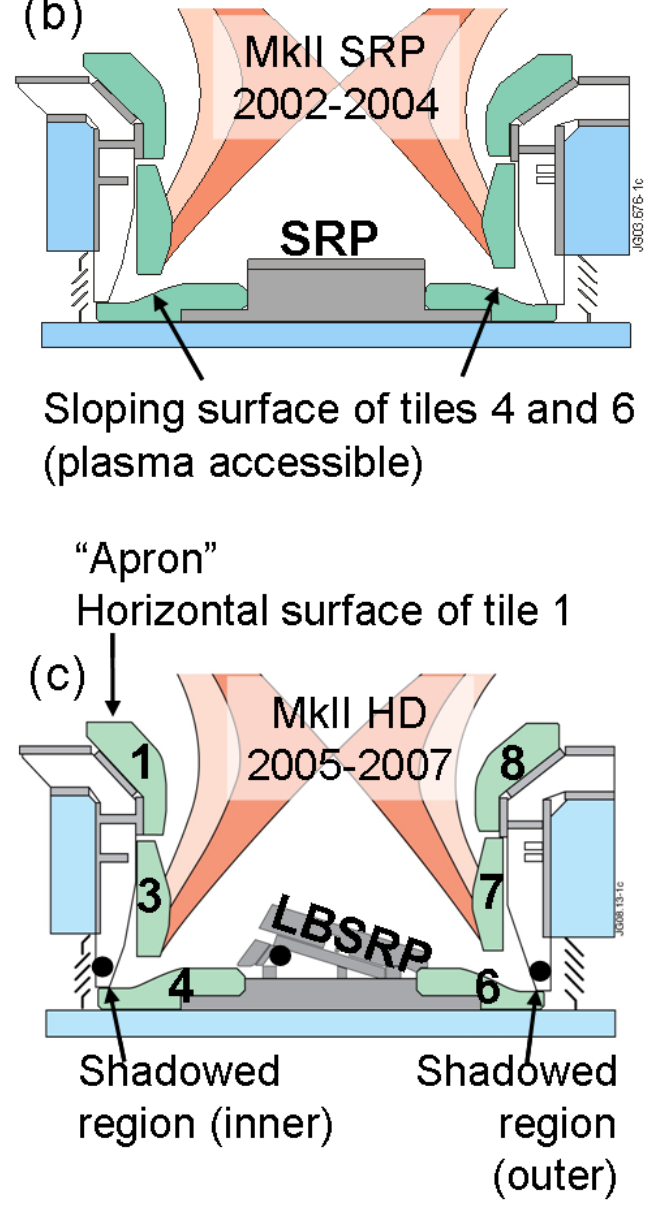

Figure 1. Divertor geometries in JET (a) 1999-2001 Mk II gas box (MkII GB) divertor, (b) 2002-2004 MkII septum replacement plate (MkII SRP) and (c) 2005-2007 the MkII high delta (MkII HD) divertor. The shadowed region at the inner and outer divertor takes in the louvres, shown as diagonally hatched lines in the bottom corner of each cross section, as well as the lower horizontal surface of tiles 4 and 6, indicated in (c). The sloping surface of tiles 4 and 6 , indicated in (b), are accessible by the plasma. The location of diagnostics installed in the divertor in 2004 are indicated in (c) by block dots $(\bullet)$

divertor came from the analysis of tiles from the MkIIA divertor [2]. In particular a tritium-rich spalling deposit was found on the inner floor tiles and the inner louvres, whereas the outer floor tiles and louvres were essentially clean with only small amounts of tritium being found. This asymmetric behaviour has continued in subsequent campaigns. For example, for the MkII GB divertor the direct measurement of tiles using a micrometer showed net deposition on the inner divertor tiles while outer divertor tiles did not [3], except for deposition on the sloping surface of the outer floor tile 6 (which is accessible by the plasma). The deposition at this location on tile 6 was similar in nature to that observed in the equivalent location on the inner floor tile 4 and was dusty and easily compressed compared with other co-deposited material. The outer divertor vertical tiles have shown net erosion over all campaigns; results such as the erosion of tungsten (W) marker coatings on tiles 7 and 8 of the MkII SRP are evidence of this $[4,5]$.

Following the installation of the MkII SRP divertor, some changes in the erosion and deposition characteristics at the inner and outer plasma-shadowed regions of the divertor have been noted. Prior to the MkII SRP, deposition on the shadowed region of the outer floor tile 6 (i.e. the lower horizontal surface shown in figure 1(c)) was minimal with a deposit of only $4.2 \mu \mathrm{m}$ being measured on the tile removed from the MkII GB divertor, compared with $87 \mu \mathrm{m}$ at the inner shadowed region of floor tile 4. For the MkII SRP, the amount of deposition in the same location has increased to $32 \mu \mathrm{m}$ at the outer floor tile 6 and $110 \mu \mathrm{m}$ at the inner floor tile 4 . Thus, the ratio of deposit on the plasma-shadowed region of the inner and outer floor tiles has decreased from $20: 1$ to $3: 1$. Post-mortem analysis of the most recent floor tiles removed from the MkII HD divertor again shows significant deposition at the outer shadowed region.

Some correlation of these changes to the pattern of erosion/deposition in JET with campaign averaged plasma configurations has been possible; however, it is clear that further detailed analysis of other divertor diagnostics, and especially of time-resolved data provided by techniques such as quartz microbalances (QMB) and rotating collectors (RCs) is required. Just as the asymmetric erosion/deposition in the 1996-1998 JET campaign caused major reassessment of plasma boundary modelling, the changes towards a more balanced deposition between the inner and outer louvre regions should provide new stimuli for modelling.

\section{Experimental details}

The MkII HD divertor was installed in 2004 and will remain in JET until the end of 2009. In 2007 there was a vessel intervention during which a poloidal set of divertor tiles was removed. Compositional analysis of the tile surfaces and their deposits has been performed using ion beam analysis (IBA) techniques including nuclear reaction analysis (NRA) as well as secondary ion mass spectrometry (SIMS). Results for NRA give atomic concentration in the first $\sim 7 \mu \mathrm{m}$ of the surface of the deposit using $2.5 \mathrm{MeV}^{4} \mathrm{He}^{+}$ions, whilst SIMS gives data profiles through the deposit although quantitative analysis is more difficult. The thickness of deposits was measured using cross-sectional optical microscopy and SIMS.

Diagnostics were installed into the JET vessel in 2004 to provide time-resolved information on deposition and erosion. The diagnostics consist of $\mathrm{QMB}, \mathrm{RC}$, mirror test units (MTU) and silicon deposition monitor details of which can be found elsewhere [6-8]. The QMBs and RCs were installed at the inner and outer corners of the divertor and under the load bearing septum replacement plate (LBSRP), as shown in figure $1(\mathrm{c})$. The QMBs enable pulse-by-pulse 
monitoring of deposition and erosion in these locations during the JET experimental campaign while the RC can provide deposition information with a resolution of 30-60 pulses, but only from post-mortem analysis. The first RCs were removed from the JET vessel in 2007 and initial detailed results from the RC located under the LBSRP are presented elsewhere [9]. In addition, two RCs and two MTUs were installed at the outer mid-plane of the JET main chamber in locations such that they were exposed to the beryllium (Be) flux from the Be evaporator head in octant 3 of the vessel. The Be evaporator heads are extended periodically into the vessel during non-operational days (usually weekends) and run for approximately $50-60 \mathrm{~min}$ to condition the vessel wall. Post-mortem analysis of these components have provided information on the Be evaporation during the 2005-2007 experimental campaigns and are presented here.

\section{Results and discussion}

\subsection{Deposition at the inner divertor}

During the JET intervention in 2007 all tiles were inspected using a camera controlled via a remote handling arm. During this inspection, a rough appearance was noted on the apron (i.e. the top horizontal surface) of all tiles 1 around the vessel. This rough appearance was due to a spalling deposit, which extended over two thirds of the apron surface, with the remaining third being deposit, which was in the region shadowed by the adjacent tile. From optical microscopy, the thickness of the spalling deposit was $119 \mu \mathrm{m}$ for a tile installed 1998-2007 and $115 \mu \mathrm{m}$ for a tile installed in 2005-2007. The first signs of spalling deposit at this location were seen on tiles that were in the vessel from 1998-2004, although not to the extent of the tiles removed in 2007: the thickness of the deposit on the apron of these tiles was $98 \mu \mathrm{m}$. It appears that $100-120 \mu \mathrm{m}$ is the critical thickness for the adhesion of films in this location, even though much thicker adherent films are seen elsewhere in the divertor (see later).

From NRA of a tile 1 removed from the MkII HD divertor, the $\mathrm{Be} / \mathrm{C}$ ratio of the deposit on the apron is 0.25 and on the front surface is typically $\mathrm{Be} / \mathrm{C} \geqslant 1$. The trends (i.e. lower $\mathrm{Be} / \mathrm{C}$ on the apron than on the front surface) are the same as the results found for the MII SRP divertor with $\mathrm{Be} / \mathrm{C}=0.5$ on the apron of tile 1 and typically $\mathrm{Be} / \mathrm{C} \geqslant 1$ on the front vertical surface. The composition of the deposits is known to be altered as a result of chemical erosion of the deposited $\mathrm{C}$ by the flux of $\mathrm{D}$ ions and neutrals on the surface of the tiles. The ratio of the $\mathrm{Be}$ and $\mathrm{C}$ impurity ions flowing to the inner divertor along the scrape-off layer (SOL) is likely to reflect the $\mathrm{Be} / \mathrm{C}$ ratio in the plasma of $\sim 0.1$. However, as a result of the chemical sputtering this ratio increases as the $\mathrm{C}$ is re-eroded and migrates to the inner shadowed region of the divertor leaving a Be-rich deposit on the inner tile surfaces [2]. This effect is less on the horizontal surface of the apron of tile 1 than on the vertical surface of tile 1, probably due to two reasons. Firstly, the apron is deeper into the SOL where surface temperatures and ion densities are lower, and secondly the pressure of neutrals is expected to decrease further away from the divertor corner.
The thickness of deposit also varies greatly between the apron and the front vertical surface of tile 1 where a thick deposit of $115 \mu \mathrm{m}$ was observed on the apron while the thickness' ranged from $49 \mu \mathrm{m}$ near the top edge of the front surface to $14 \mu \mathrm{m}$ at the bottom edge, for the tile installed from 2005 to 2007. This may be due to higher fluxes on the apron resulting from the angle of incidence between the field lines and surface giving a larger 'wetted area' in the poloidal direction. As a consequence the rate of deposition is expected to be greater on the apron than on the front vertical surface of tile 1.

In general, the $\mathrm{Be} / \mathrm{C}$ ratios for tiles 1 and 3 were not markedly different from that observed for the MkII SRP tiles. This is surprising as the installation of the MkII HD divertor has allowed high-delta plasma configurations with the inner strike point high on tile 3 and on tile 1, as shown in the inset in figure 4, whereas previously most inner strike points were on the lower part of tile 3 . In the high-delta configuration the top of tile 3 experiences higher surface temperatures, incident $\mathrm{C}$ and Be flux and plenty of deuterium (D) for chemical sputtering, whereas the bottom of the tile is in the private flux region with lower surface temperatures, no incident Be and lower D fluxes. For this reason one might expect markedly different characteristics to be observed from the analysis of the inner divertor tiles between the MkII HD and MkII SRP tiles, however this was not the case. In figure 2 the distribution of the strike-point position is plotted using the s-coordinate system which follows the contour of the surface of the tiles. This scale has been adopted for the MkII HD divertor; however, for ease of comparison, data for the MkII SRP divertor have been re-plotted on equivalent s-coordinates positions. This plot shows only a small increase in the frequency of strike-point location on tile 1 and the top of tile 3 , which is not significant in terms of the total operating time. Thus, the fact that the $\mathrm{Be} / \mathrm{C}$ ratios for tiles 1 and 3 for the MkII HD divertor were similar to those for the MkII SRP divertor is reasonable.

\subsection{Deposition on the divertor floor tiles}

Deposits from the plasma accessible sloping surface and plasma inaccessible shadowed region of the inner and outer floor tiles, (tiles 4 and 6 as shown in figure 1(c)), from different divertor campaigns have been measured by cross-sectional optical microscopy. Direct measurements of tiles for the MkII GB and MkII SRP campaigns for tiles installed from 1998-2001 and 2002-2004, respectively are available; however, the thickness of deposit for the MkII HD divertor geometry from 2005-2007 has been obtained indirectly by subtracting the previous campaign values from the results for a tile which was in the vessel from 1998-2007. Based on these optical microscopy results and assuming that the deposit was a result of divertor plasmas, the rates of deposition were determined. In the case of the outer floor tile 6 the deposition rate on the sloping plasma accessible surface was found to increase by a factor 1.5 from $2.3 \mathrm{~nm} \mathrm{~s}^{-1}$ in the period 1999-2001 to $3.4 \mathrm{~nm} \mathrm{~s}^{-1}$ for the period 2005-2007. The increase in deposition rate in the shadowed region of tile 6 was markedly higher, rising by a factor $\sim 6$ from 0.06 to $0.34 \mathrm{~nm} \mathrm{~s}^{-1}$. The results for the inner divertor are less clear 


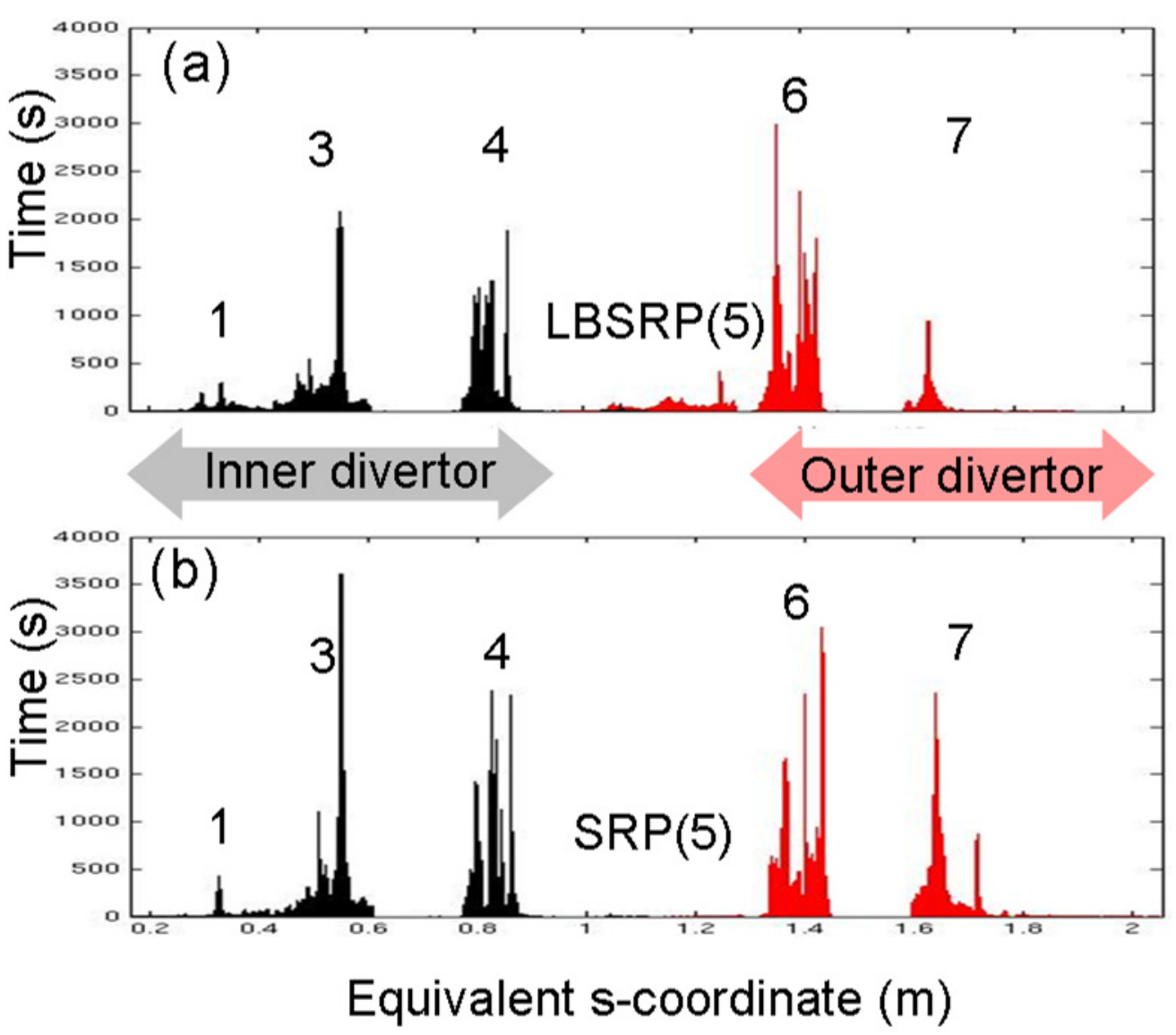

Figure 2. Distribution of strike-point positions verses time from magnetic configuration data. (a) 2005-2007 MkII HD divertor and (b) 2001-2004 MkII SRP divertor. Divertor tile numbers are indicated. The data are plotted using the s-coordinate system, which follows the contour of the surface of the divertor tiles with the origin being at the inboard corner of the high field gap closure tile inboard of tile 1. This scale has been adopted for the MkII HD divertor; however for ease of comparison, the data for the MkII SRP divertor have been plotted using equivalent s-coordinate positions where the coordinates for the outer divertor start $\mathrm{s}=1321 \mathrm{~mm}$ (see figure 3 ).

as there was a overall decrease in the thickness of deposit on the sloping surface of tile 4 deduced from the 1998-2007 tile samples (i.e. the sum of the deposits for 1998-2001 and 2002-2004 exceed that for the tiles from 1998-2007). Until further tiles due to be removed from the JET vessel in the 2009-2010 intervention are analysed, it is uncertain if this is an anomalous result or whether a net erosion has occurred in this region. In the shadowed region of the inner divertor floor tile 4 an overall increase in the rate of deposition has been observed, from $1.3 \mathrm{~nm} \mathrm{~s}^{-1}$ in 1999-2001 to $2.4 \mathrm{~nm} \mathrm{~s}^{-1}$ for the tile removed in 2007. This is indicative of a relative increase in deposition at the outer divertor leg and a reduction in the degree of asymmetry between the deposition at the inner and outer divertor compared with that observed prior to the MkII SRP divertor.

Deposition was also observed on the outer divertor carrier onto which the outer vertical divertor tiles are mounted. In 2007 when a selection of divertor carriers was removed from the vessel, flaking deposits were observed on the ribs of the outer divertor carrier, located just in front of the louvres shown in figure 1. A similar flaking deposit was also observed on the inner carrier ribs, although the deposit at the outer divertor was much thinner than the deposit at the inner divertor (as expected) thus indicating that it was more stressed.

The deposit on the outer sloping surface of tile 6 , in vessel from 1998-2007 has reached $800 \mu \mathrm{m}$ in 1998-2007.
This is the thickest deposit observed to date on JET divertor tiles and is still well adhered to the surface. The deposit structure as seen in cross sections appeared dense, in contrast to the deposit on the sloping surface of tiles 4 and 6 from the MkII GB divertor where films were reported as being soft when thickness measurements were made using a micrometer [3]. This could be due to the difference in composition or alternatively different plasma conditions on these tiles, although no correlation has been established at this time.

The composition of the deposits on the inner and outer floor tiles has also changed for the MkII HD compared with previous campaigns. Figure 3 shows the distribution of the $\mathrm{Be} / \mathrm{C}$ ratio for tiles 4 and 6 removed from the JET vessel after the last three JET operating periods (MkII Gas Box, MkII SRP and MkII HD divertors) using the s-coordinate system. Be/C ratios are plotted, rather than the Be concentration, to reduce errors in the results associated with variation in mounting tiles at different times. The Be/C ratios are determined from NRA which analyses to a depth of $\sim 7 \mu \mathrm{m}$; however SIMS data confirms uniform concentration of Be to depths up to $80 \mu \mathrm{m}$, such as on the top edge of the sloping section of tile 6.

The results show an increase in the $\mathrm{Be} / \mathrm{C}$ ratio on the sloping surface of the 2005-2007 tiles. The increase in the amount of $\mathrm{Be}$ is particularly noticeable for tile 6 . The ratio 


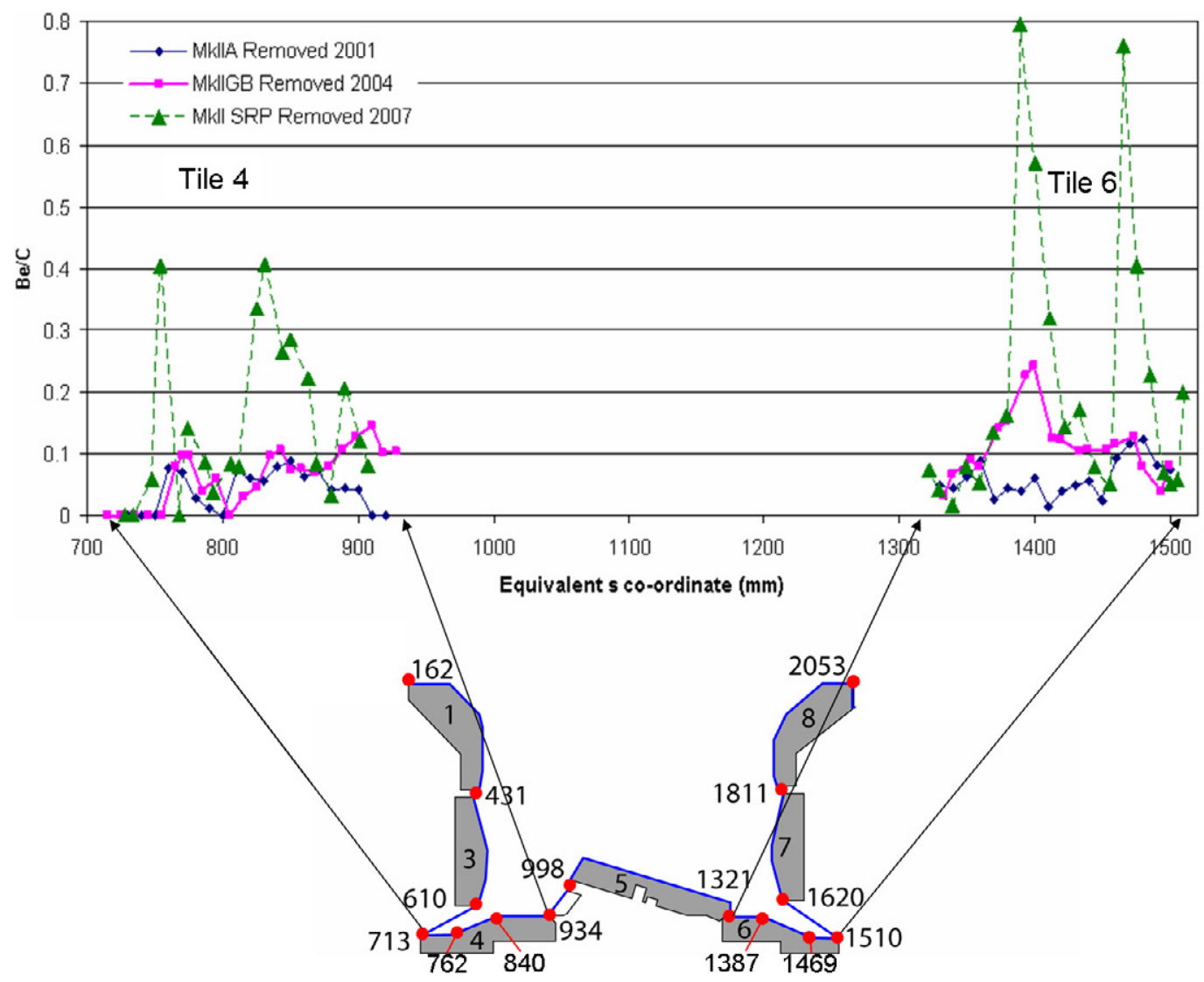

Figure 3. Be/C ratio distribution for inner and outer divertor floor tiles (tile 4 and tile 6, respectively) for tiles from the MkII GB, MkII SRP and MkII HD operating periods, plotted using the s-coordinate system adopted for the MkII HD. Below is a cross section showing the full s-coordinate system adopted for the MkII HD divertor geometry. For ease of comparison, equivalent s-coordinate locations have been used to plot the data on tile 6 for the MkII GB and MkII SRP divertor geometries.

has increased from $<0.1$ for a tile removed in 2001 up to 0.8 for a tile removed in 2007 at some locations comparable to values seen on the surface of tiles 1 and 3 above. Similar increases are seen on tile 4 where the Be/C ratio has increased from $<0.1$ up to $\sim 0.4$ in some regions for tiles removed in 2001 and 2007, respectively. Chemical sputtering of C, as discussed in sections 3.1 , is also likely to occur on tiles 4 and 6 , so the ratio of $\mathrm{Be} / \mathrm{C}$ incident at these regions must have been significantly lower than for the inner divertor tiles. For the inner divertor the $\mathrm{Be} / \mathrm{C}$ ratio was similar for the MkII GB and MkII SRP divertors with the increase in the Be being observed only for the most recent MkII HD divertor tiles; however, for the outer divertor tile there is an increase in Be concentration observed at the top of the sloping surface of tiles from the MkII SRP divertor, with a marked increase on the 2007 tile. The structure of the Be concentration shows some correlation with the distribution of the strike-point positions for the MkII SRP and MkII HD divertor geometries as shown in figure 4 . That is to say that the peaks roughly correlate between the strike-point distribution and the $\mathrm{Be} / \mathrm{C}$ ratio data. There is however some discrepancy between the two sets of data, this could be due in part to the allocation of the s-coordinate for the NRA measurement point for the $\mathrm{Be} / \mathrm{C}$ ratio, which could be $\pm 3-4 \mathrm{~mm}$. An additional source of discrepancy is the use of the magnetic configuration to determine the strike-point distribution, as discrepancies of $10-20 \mathrm{~mm}$ between the strike-point allocation determined from magnetic configuration and from the infrared camera images of the tiles in the divertor have been observed. The magnetic strike point does not indicate the energy or flux distribution on the tiles, which might offer an even better correlation with the $\mathrm{Be} / \mathrm{C}$ ratio. While the structure seen in the amount of $\mathrm{Be}$ may correlate with the strike-point position the increase in Be concentration is not understood. Campaign averaged spectroscopy data indicates that the $\mathrm{C}$ and Be levels in the main chamber are similar for the MkII GB, MKII SRP and MkII HD divertors as shown in figure 5. This suggests that the amount of Be has not increased as an impurity source over the periods being compared. In addition, the number of beryllium evaporations has decreased over the period since 1998 as the condition of the vessel has improved; in earlier operating periods Be evaporations were carried out twice weekly to condition the vessel, this has now reduced to intervals greater than a week in the MkII HD operating period. A possible additional source of Be in the MkII SRP and MkII HD divertors compared with the MkII GB divertor could be due to the sputtering of deposits from the (inner) divertor by Type I ELMs [10]. Such material transport was not possible with the MkII GB divertor geometry. However material transport across the private flux region is still the subject of much debate.

For the MkII HD divertor the $\mathrm{D} / \mathrm{C}$ ratio remains fairly constant on the plasma accessible surfaces of tile 6 and rises by a factor $\sim 10$ in the plasma shadowed horizontal surface. 


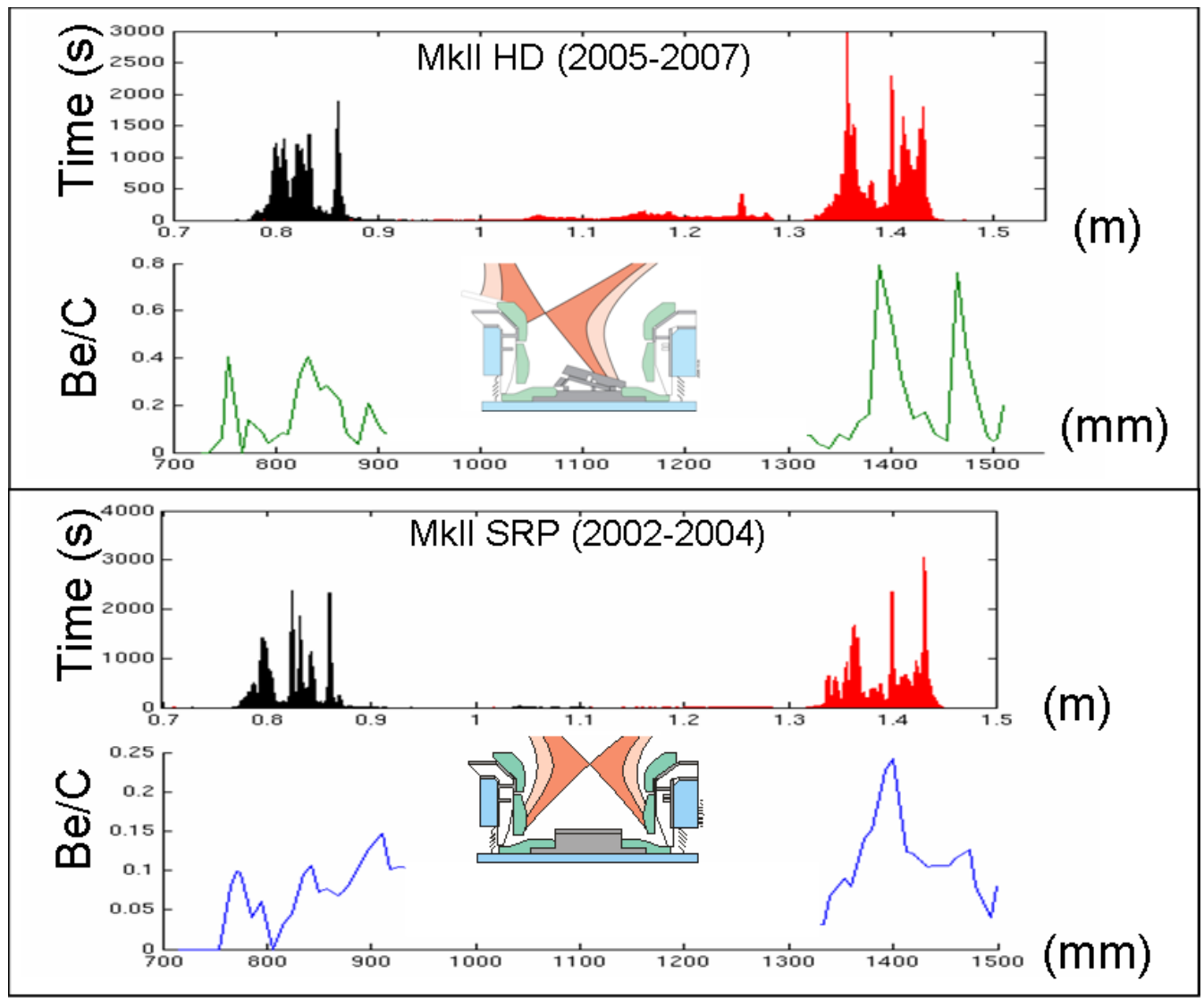

Equivalent s-coordinate

Figure 4. Comparison of the strike-point position and $\mathrm{Be} / \mathrm{C}$ ratio on inner and outer divertor floor tiles.

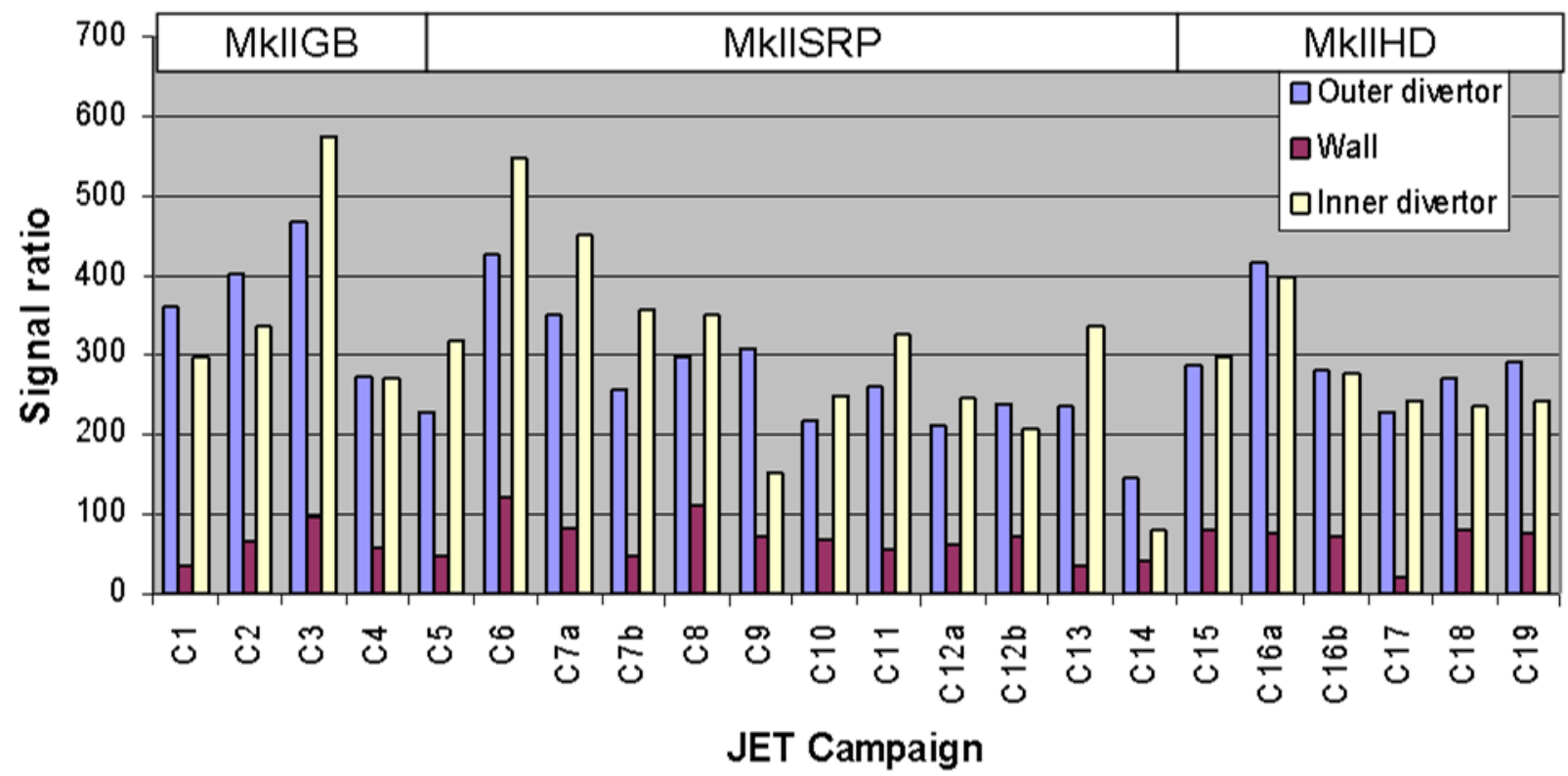

Figure 5. C III/Be II impurity flux ratios for the inner divertor, outer divertor and main chamber. The period of installation of each divertor geometry is indicated at the top of the figure.

On tile 4 a similar trend is observed, with an additional increase in the $\mathrm{D} / \mathrm{C}$ ratio at the corner of the tile closest to the LBSRP (i.e. s-coordinate $=900-934 \mathrm{~mm}$ in figure 3). Similar trends were observed in the earlier campaigns. No correlation is observed between the $\mathrm{D} / \mathrm{C}$ ratio and $\mathrm{Be} / \mathrm{C}$ ratio for these tiles.

\subsection{Erosion/deposition at the outer divertor}

In the MkII SRP campaign erosion of $\mathrm{W}$ coatings was observed giving direct evidence for net erosion at the vertical outer divertor tiles [4]. The erosion has continued in this region for the MkII HD divertor. SIMS measurements indicate 
only a very thin layer $(<1 \mu \mathrm{m})$ near the bottom and near the centre of the surface of tile 7 and from photographs of tile 7 some changes in colour due to thickness fringes from very thin layers can be seen; however, the CFC fibre planes are still evident showing that the tile surface is essentially clean. Thus erosion processes are still dominating over deposition processes in this region of the divertor.

\subsection{Time-resolved diagnostics}

Clearly from discussions in sections 3.2 , it is difficult to show the effects of individual plasma configurations on erosion, deposition and material transport from campaign averaged plasma parameters and post-mortem analysis. Thus, much of the effort in identifying the effects of individual plasmas has focused on results from a series of deposition monitors installed in JET as detailed in section 2, the results of which are discussed below. Once the effects of individual plasmas configurations are better understood, extrapolation to integrated post-mortem analysis may become possible.

Details of measurements with the QMBs during the 2005-2007 operational period have been reported elsewhere [11-13]. In general terms, the amount of deposition on the QMB sited at the inner corner near the inner louvres of the divertor was greatest when the strike point was moved to be on tile 4, and increased nonlinearly with ELM energy. The QMB sited at the outer corner near the outer louvres of the divertor received small amounts of deposit in some pulses, and the deposit on its sensing crystal experiences a small amount of erosion during other pulses; the net deposition was close to zero, and attempts to correlate the behaviour with plasma parameters are proving difficult but are continuing [11]. The QMB in the LBSRP unit which faces the inner divertor tiles showed two clear periods of 'integral deposition', separated by a period of 'integral erosion', with another period of erosion in progress when the QMB exceeded its operating limit. The periods of 'integral deposition' and 'integral erosion' correlate with operational periods when the strike points were normally of tiles 3 and 4, respectively [12]. A correlation has been made of the deposition/erosion data from the QMB in the LBSRP with deposition on a RC at a similar location [9]. The results showed that the QMB only collects $\sim 25 \%$ of the deposition collected at the RC. This suggests a difference in surface temperatures being reached during the plasma pulse between the RC Si disc and the QMB crystal.

As mentioned in section 2, RCs were mounted at two positions at the outer mid-plane; in each case the RC shared a bracket with a MTU [6]. One of the RC units was close to a Be evaporator head, and the slit $(2 \mathrm{~mm}$ wide) was aligned with the evaporator so that the full Be flux would impinge on the collector. Since the Be evaporated normally deposits on carbon surfaces within JET, in order to provide a calibration for the flux from the evaporator, the collector at this location was made of carbon, whereas all other RC collectors are made of polycrystalline silicon. A photograph of the collector after removal in 2007 is shown in figure 6.

It can be seen in figure 6 that the collector only rotated through about $100^{\circ}$. The reason why the rotation stopped is unknown, however stripes from four separate evaporations

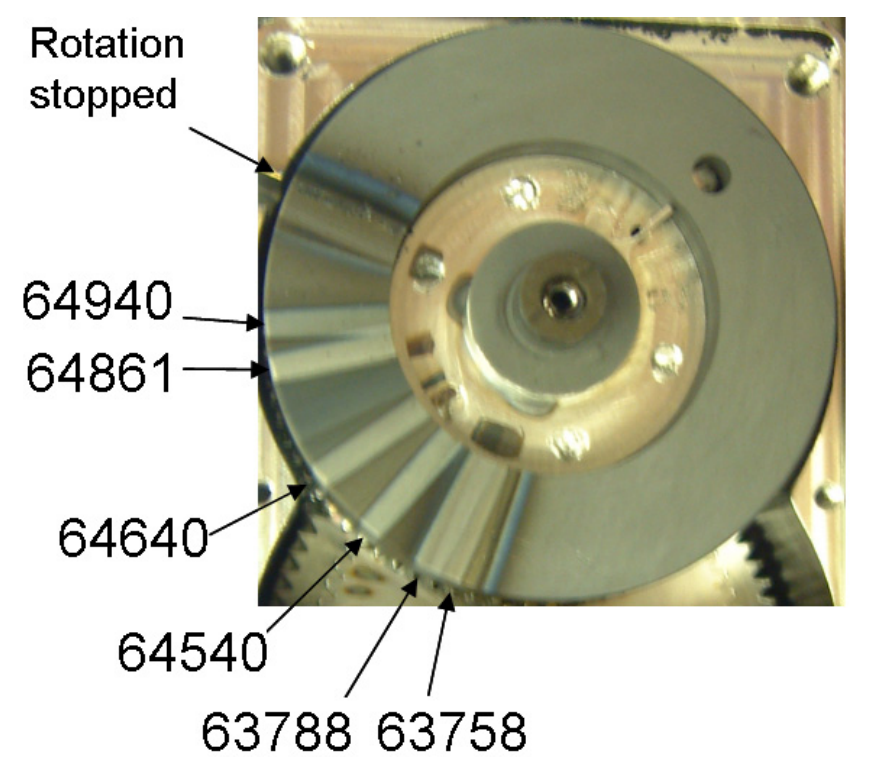

Figure 6. Carbon disc removed from RC located at the outer mid-plane of the JET main chamber. Bright stripes are correlated with deposition from the beryllium head in octant $3 \mathrm{E}$ at the outer mid-plane.

and a double stripe at the start are clearly visible; there is also a thick band where the rotation stopped. The first pulses after each of these six evaporations are 63758, 63788, $64540,64640,64861$ and 64940. There were a total of $84 \mathrm{Be}$ evaporations from this head during the 2005-2007 operational period, so there is a much thicker deposit in the area exposed after the rotation stopped (which was exposed to 78 evaporations). The amount deposited in each of the six identifiable stripes varies considerably-the average of the first two is $2.2 \times 10^{18} \mathrm{Be}$ atoms $\mathrm{cm}^{-2}$, the average of the next four is $0.25 \times 10^{18} \mathrm{Be}$ atoms cm $\mathrm{cm}^{-2}$ (but varies within this sub-set by a factor of four), and the amount at the end point is $14.4 \times 10^{18} \mathrm{Be}$ atoms cm $\mathrm{cm}^{-2}$ (that is, an average of $\sim 0.2 \times 10^{18}$ Be atoms $\mathrm{cm}^{-2}$ for each of the 78 contributing evaporations). Although some other deposits do appear to be present between the Be evaporation stripes, as seen in figure 6 , since deposited carbon cannot be differentiated from the carbon substrate, no useful results were obtained from IBA of these deposits.

When the Be heads are inspected during a shutdown they are invariably covered with an oxide film, and it is standard procedure to clean the heads during shutdown, as was done in the 2006-2007 shutdown. It would appear from this analysis that the efficiency of the head dropped off by almost an order of magnitude after the first two evaporations and thereafter remained reasonably constant. This is consistent with the scenario in which after the initial evaporations the head becomes covered with an oxide film, and the efficiency of evaporation is then limited by the diffusion of Be atoms to the surface through the oxide film. The side of the MTU adjacent to the RC facing towards the $\mathrm{Be}$ evaporator was also covered with $\mathrm{Be}$, and may be expected to have experienced a similar total flux of Be. The mean of two analysis points on the MTU was $21.1 \times$ $10^{18}$ Be atoms $\mathrm{cm}^{-2}$, which compares well with the total deposition on the RC collector of $20.8 \times 10^{18} \mathrm{Be}$ atoms cm${ }^{-2}$. 
However, it should also be noted that the exposed end point of the collector and the side of the MTU would have been exposed to bombardment by charge exchange neutral atoms throughout all (or nearly all, in the case of the collector) the operational period, so some of the Be film may have been eroded and agreement with the mean of the four deposits that were largely protected from this erosion may be fortuitous.

\section{Conclusions}

Post-mortem analyses of tiles removed from the different divertor geometries have provided empirical data showing the asymmetry of deposition/erosion at the inner and outer divertor legs. However, the most recent results from tiles removed in 2007 indicate that more deposition is occurring at the shadowed region of the outer divertor, reducing the degree of asymmetry between deposits found at the inner and outer JET divertor legs. In addition, the Be concentration of deposits on the plasma accessible surfaces of the inner and outer divertor floor tiles has increased. While the local distribution of the Be can be correlated with average strike-point position, the transport of $\mathrm{Be}$ to these tiles and the increased deposit at the outer divertor leg has not so far been explained by campaign averaged plasma configuration data. This shows that the time-resolved data provided by diagnostics located in the vessel play an important role in conjunction with post-mortem analysis in understanding material migration. For example, the QMB and $\mathrm{RC}$ diagnostics have shown that the balance between deposition and erosion is strongly dependent on plasma configurations, particularly under the LBSRP and at the outer divertor.

\section{Acknowledgments}

This work was funded jointly by the UK Engineering and Physical Sciences Research Council and by the European Communities under the Contract of Association between EURATOM and UKAEA. The views and opinions expressed herein do not necessarily reflect those of the European Commission.

Published under licence from EURATOM.

\section{References}

[1] Coad J P et al 1999 Phys. Scr. T81 7

[2] Coad J P et al 2001 J. Nucl. Mater. 290-293 224

[3] Coad J P et al 2003 J. Nucl. Mater. 313-316 419-23

[4] Mayer M et al 2007 J. Nucl. Mater. 363-365 101

[5] Coad J P et al 2007 J. Nucl. Mater. 363-365 287

[6] Coad J P et al 2005 Fusion Eng. Des. 74745

[7] Esser H G et al 2007 J. Nucl. Mater. 363-365 146-51

[8] Rubel M et al 2006 Rev. Sci. Instrum. 77063501

[9] Coad J P et al 2009 Phys. Scr. T138 014023

[10] Huber A et al 2009 J. Nucl. Mater. 390-391 830-4

[11] Kreter et al 2009 J. Nucl. Mater. 390-391 148-51

[12] Esser H G et al 2009 J. Nucl. Mater. 390-391 148-51

[13] Kreter A et al 2009 Phys. Rev. Lett. 102045007 